\title{
The diagnosis of mandibular assymmetries
}

\author{
BRIONNE Camille, CADRE Bernard, \\ LAROCHE Yann, LHOTELLIER Jessy, \\ MAZE Morgan, RAFFRE Alexis, SOREL Olivier
}

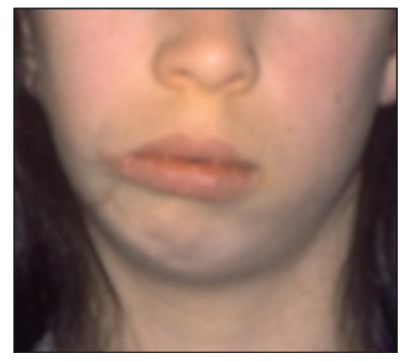

\section{ABSTRACT}

Asymmetries are found in every living being. A non-symmetrical structure is not necessarily pathological. Why then, is the diagnosis of asymmetry relevant for us as practitioners? In orthodontics, we can anticipate difficulties with treatment if we are well informed about the principles of symmetry and thoroughly familiar with craniofacial growth.

Severe mandibular asymmetry is relatively rare, appears early and becomes apparent at first sight to the practitioner. We find it associated with syndromes in patients for whom early multidisciplinary treatment is indicated.

Dyssymetry is a defect rather than an absence of symmetry. It is a mild form of asymmetry where the line between pathological and nonpathological is still unclear. The face can present different types of dyssymmetries including the whole gamut ranging from dental imbalance to imbalances of the jaws to cranial imbalances, each with its own signature that leaves its characteristic mark on the face. When dealing with mandibular laterodymorphias, the diagnosis must be made early in order to initiate an individualized treatment plan. We are providing some summary fact sheets that make it possible for the practitioner to clearly distinguish between the principal mandibular anomalies.

\section{KEY WORDS}

Dyssymmetry

Asymmetry

Mandible

Diagnosis 

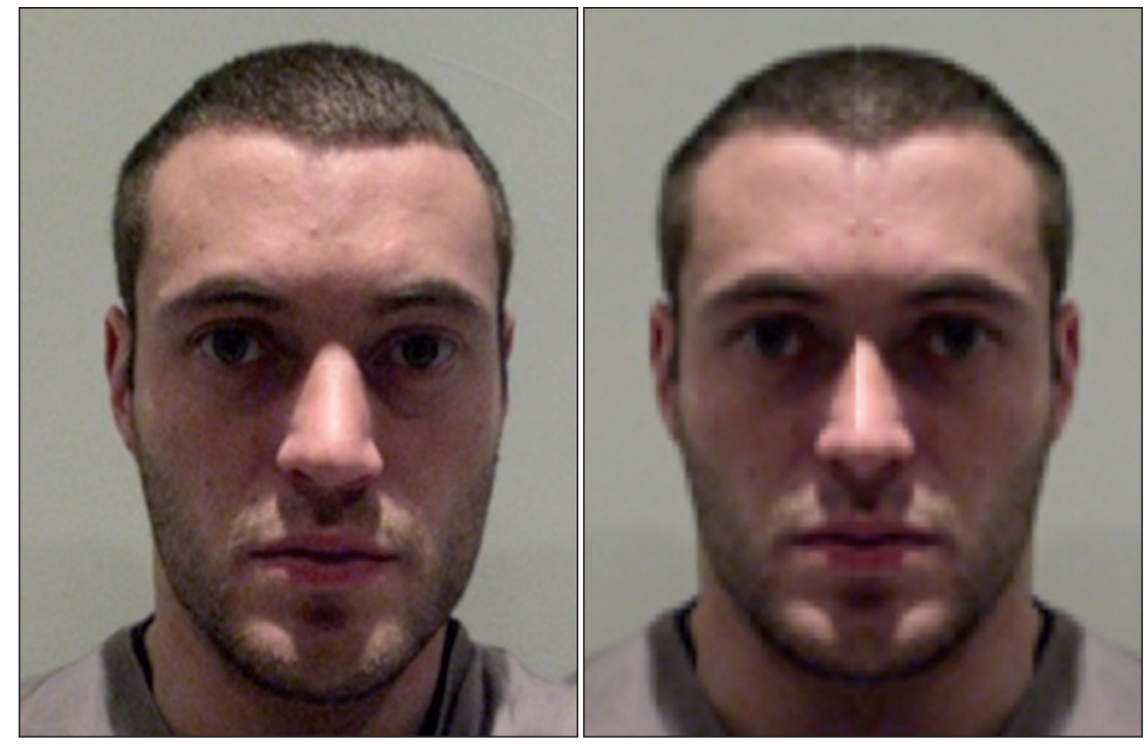

Figure 1

Photographs and chimerisms of Mr. R.

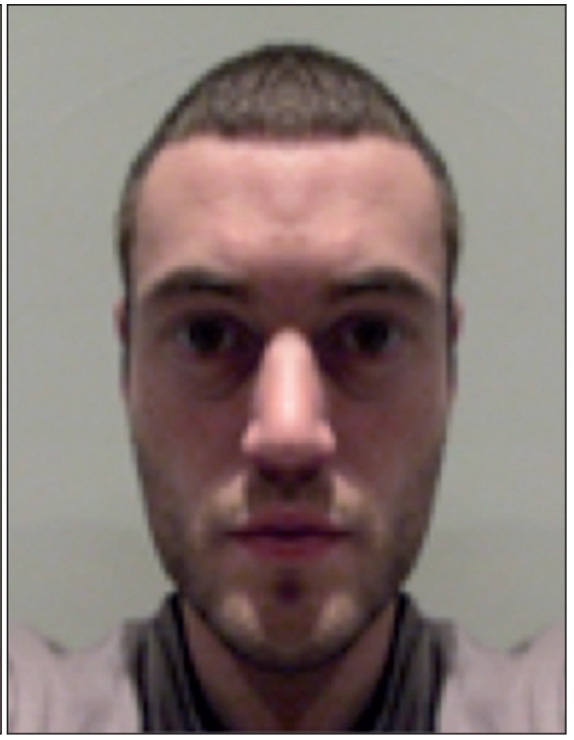

Geometry defines symmetry as a concordance of shape, size and position in relation to an axis, a point or a plane. Therefore, symmetry is a mathematical concept that is applicable to the living world. However, a number of artists have displayed dyssymmetries in their works or their models: in 1887, Hasse, in his writings on Ancient Greek Statues had already pointed out slight dyssymmetries exhibited by the Venus de Milo. It would seem that dyssymmetry rather than symmetry is a characteristic of human beings (Fig. 1).

According to Cohen, an asymmetric structure is completely normal in our development: this defect is found in the skeleton (clavicles, ribs, fibula) as well as on facial photos when the face is divided along the median line and two very different chimerisms are obtained. Therefore, if dyssymmetry is normal in human development, in facial growth and growth in the rest of the body, why is it important to diagnose it in our patients?

In the discipline of orthodontics, we have to accurately establish the standards for what is considered normal, because these defects are not always obvious and strictly speaking, rarely constitute pathologies. The continuing goal of our treatments is to make the life of the patient better by improving interdental and maxillary functions as well as facial esthetics; however, orthodontics alone is insufficient when the required compensation necessarily becomes iatrogenic.

A solid diagnostic approach, based on a set of norms, makes it possible for the practitioner to anticipate difficulties during treatment. In other words, the question that arises is the distinction between normal 
dyssymmetry and the point after which dyssymmetry becomes pathological. The other question is to de- termine the causes in cases where there is a lack of symmetry.

\section{1 - ASYMMETRY AND DYSSYMMETRY}

\section{1 - 1 - Asymmetry}

The term "asymmetry" literally means "lack of symmetry". In our discipline, true asymmetry is relatively rare. These dysmorphias appear very early during the developmental period. Our focus here will be limited to the study of mandibular asymmetry.

Mandibular asymmetry has an embryological origin. The morphogenesis of the facial bones is the culmination of complicated mechanisms that take place between the 4th and 10th week in the life of the embryo. A malfunction of the mechanisms of cellular migration, of apoptosis or of cellular differentiation can compromise these processes and lead to malformations.

In the fetus, the mandible appears in the shape of two hemimandibles, consisting of mesenchymal and cartilaginous tissue, that fuse at the midline following birth. The asymmetry of the mandible is mostly encountered in patients suffering from major syndromes associated with malformations.

\subsubsection{Unilateral defects caused by insufficient condylar cartilage}

When condylar cartilage is exposed to certain pathogenic agents during the growth period, it can lead to defects in the growth of the affected condyl. The resulting mandibular asymmetry is encountered in the following cases:

- Necrotizing osteitis,

- Early exposure to radiation,

- Temporomandibular ankylosis,

- Condylar agenesis or condylar hypoplasia that will be discussed later.

The clinical picture is characterized by a deviation of the chin on the affected side. The entirety of the face is deformed: the median saggital plane is curved and the horizontal planes convergent toward the affected side. There are, in addition, a whole range of functional problems in this scenario that limit mandibular movements (Fig. 2).

\subsubsection{Otomandibular syndromes}

Otomandibular dysplasia is a congenital defect characterized by hypoplasia that involves the temporomandibular and pterygomandibular complexes to varying extents. The clinical expression of this syndrome varies and can affect the ear, the maxilla and the mandible to differing degrees.

Unilateral forms of these syndromes are generally nonhereditary and predominantly mandibular, (the mandible is affected in $89 \%$ to $100 \%$ of the cases). Mandibular dysmorphia can range from a simple morphological alteration of the condyle to the complete absence of the mandibular 


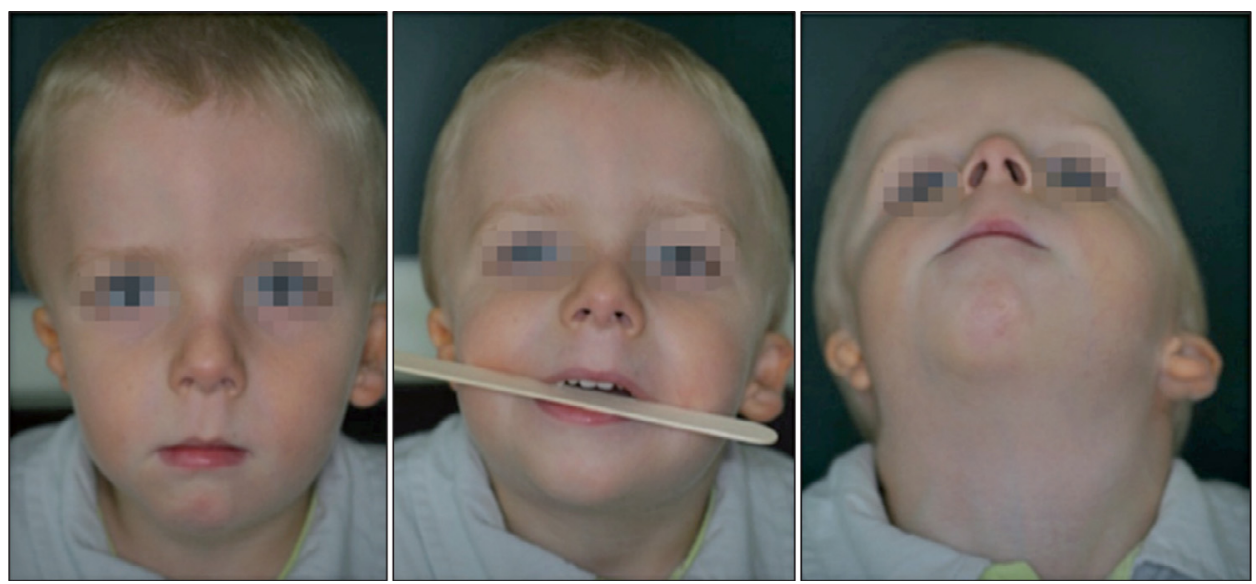

Figure 2

Mandibular asymmetry due to proliferation of the right condylar cartilage. Case of Dr. CADRE.

ramus. Condylar involvement is for the syndrome. The deficit in growth depends on the degree of severity (Fig. 3).

The healthy side compensates for the deficit in growth by an elongation of the body of the mandible and by an increase of the angle of the mandible. The chin is deviated toward the affected side. For Polley and Rune, the degree of asymmetry is constant during the growth period. Other authors such as Kearns support the hypothesis that asymmetry is progressive and worsens over time.

Along with mandibular asymmetry, these syndromes can be associated with other osseous craniofacial, cutaneous, muscular and neural anomalies or missing salivary glands. Some cerebral or extrafacial anomalies have been noted in certain cases.

When the involvement is unilateral, an isolated mandibular malformation

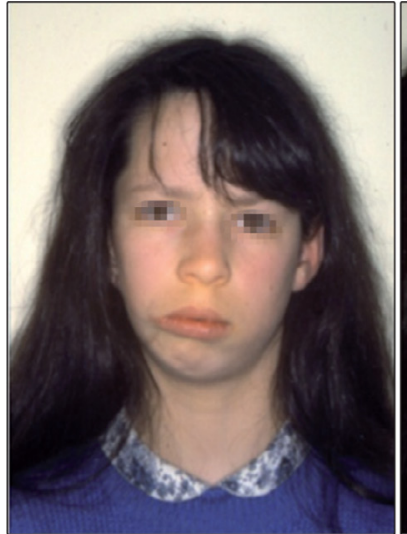

Unilateral oto-mandibular syndrome. Case of Dr. CADRE. 


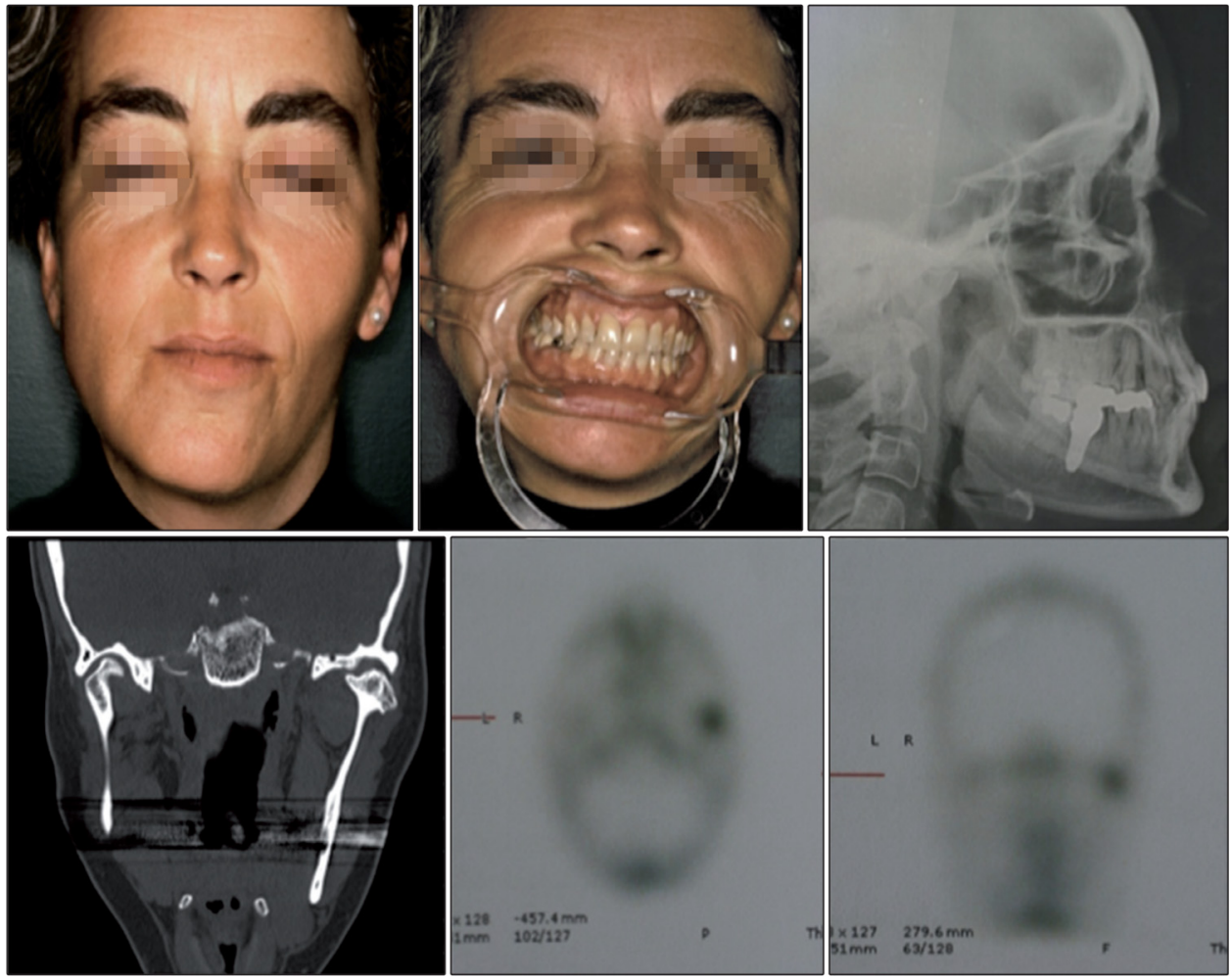

Figure 4

Left side condylar hyperplasia along with lateral head film. MRI images of two adult bone scans. Case of Dr. CADRE.

leads the practitioner to make a diagnosis of mandibular dysplasia. If, in addition to this malformation, there are other dysmorphias, the diagnosis of an otomandibular syndrome should be considered (Bettega).

\subsubsection{Defects caused by excessive unilateral proliferation of condylar cartilage}

Presently, the triggering mechanism for condylar hyperplasia is still poorly understood (Fig. 4).

Cases of condylar hyperplasia that develop later during adolescence, are a source of problems for the orthodontist. They lead to significant occlusal discrepancies.

Mandibular growth continues until the age of 21 for males. In adults, the condylar cartilage is reduced to a thin layer of cells. In cases of condylar hyperplasia, there is excessive condylar volume on the affected side. There are two hypothethical explanations for this phenomenon: either a resumption in bone growth or adaptive secondary growth. For Delaire and Petrovic, who are quoted by Aknin (2007), this problem is due to an error in interpreting the signals for 
growth stimulation. Condylar hyperplasia has a hereditary factor.

Clinically, the chin deviates towards the healthy side. The height of the mandibular ramus is greater on the affected side and gaps are prone to appear, as well as hyperprotrusion of the healthy side along with laterocclusion and slanting of the occlusal plane. A bone scan image makes it possible to demonstrate the resumption of growth of the affected condylar cartilage.

\subsubsection{Plagiocephaly}

Plagiocephaly is a generic term that describes a defect characterized by a torsion of the craniofacial complex. This terminology does not take into account the etiology of the malformation (Netherway et al. 2006).

Plagiocephaly can be caused by a mechanical problem: inappropriate forces applied during the early stages of craniofacial growth: a problem occurring during childbirth, in utero for twins, congenital torticollis, the sleep position or neuromuscular dysfunction.

Plagiocephaly can be the consequence of a premature synostosis: early unilateral synostosis of the coronal suture (an incidence of 1/10000 births) and less frequently, fusion of lambdoid suture. Treatment varies depending on the etiology; nonsurgical in the first case, surgical in cases of premature syntoses.

\subsubsection{Unilateral facial clefts (unilateral labial-alveolar-palatal clefts)}

They are the result of a dysfunction that takes place during the formation of the primary palate (between the 5th and 7th week). Macroscopically, they are caused when there is a partial or complete failure of the internal nasal buds and the maxillary bud to fuse together (Fig. 5). During normal development, the ectodermal cells in the fusion area of the buttons (wall of
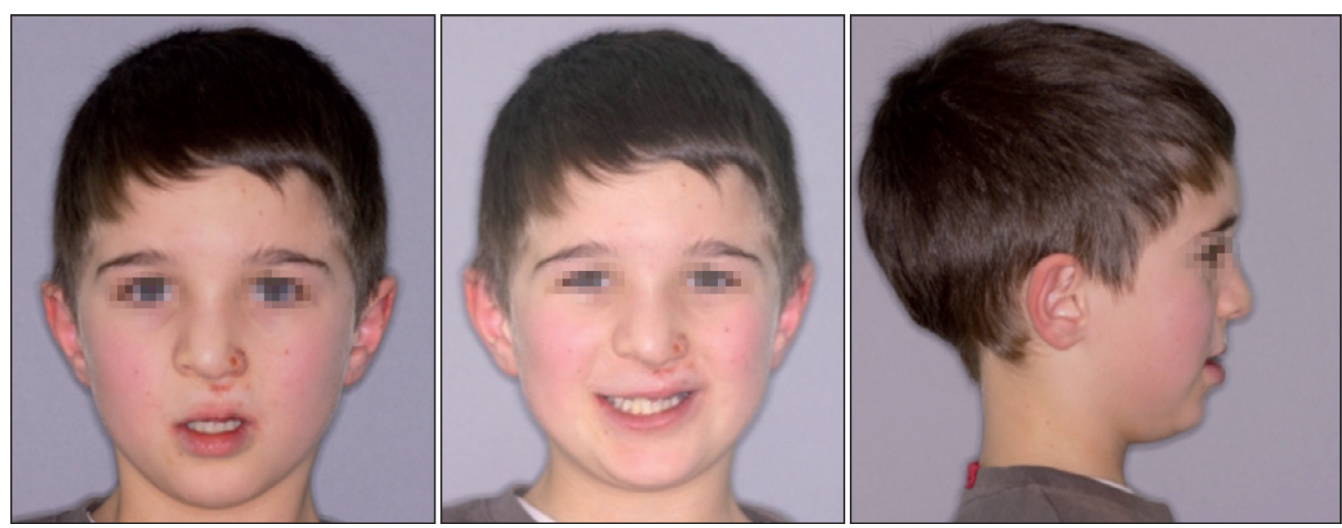

Figure 5

Unilateral facial cleft. Case of Doctor CADRE. 
Hochsetter) initiate apoptosis and are phagocytized by the underlying mesenchymal cells (Ducreux). In certain cases, this mechanism does not take place and a labial, labial-alveolar, or labial-aveolar-palatal cleft appears. The molecular and cellular agents that potentially trigger the genesis of facial clefts are numerous. Some loci where recurrent mutations occur have been isolated in the affected patients. These affected areas that encode for the signaling pathways, could explain the disturbances that occur in the formation of the primary palate. The complexity of these mechanisms explains the relative frequency of these defects $(1 / 1000$ births for labial-alveolar-palatal clefts). We differentiate between nonsyndromic clefts (isolated) and the syndromic types that present as a part of a polymalformative syndrome.

The authors attribute this to an adaptation to maxillary asymmetry, to genetics or even as a consequence of a change in fetal and then post-natal breathing dynamics (Laspos, Talmant). Therefore, an early and individualized therapeutic treatment plan seems to be indicated in order to limit this dysmorphia.

\subsubsection{Congenital anophthalmia}

By definition, congenital anophthalmia is the absence of the ocular globe as when the primary optic vesicle fails to form during embryogenesis. This is true of congenital anophthalmia. It is mostly unilateral and is frequently associated with other congenital malfor- mations. The etiology is most frequently ascribed to chromosomal abnormalities (DUFIT).

The regression of the optic vesicle during the course of embryogenesis leads to extreme microphthalmia. The clinical picture closely resembles true anophthalmia, and as described in the literature it is known as clinical anophthalmia. Sonography, scanners and MRls show an outline of an ocular globe and the optic nerve. With these dysmorphias, the ocular globe no longer induces or shapes the formation of the orbit. As a result, there is functional and significant esthetic damage that occurs in the contiguous craniofacial structures. Treatment consists in placing a prosthetic expandable orbital implant. The result is often disappointing (DUFIT).

\subsubsection{Conclusions concerning asymmetries}

Patients who present such malformations such as these can benefit from an early multidisciplinary treatment plan that starts soon after birth. Asymmetry is flagrant and the practitioner notices it at first sight. As a specialist, the orthodontist must synergistically work with the medical team to provide individualized care adapted to the abnormal appearance of the faces of these children who are confronted by the stares of others. Making a diagnosis and having a thorough understanding of these malformations are necessary requisites for planning an effective treatment plan. 


\section{1 - 2 - Dyssymmetries}

According to the Robert's Dictionary, dyssymmetry is a defect of symmetry rather than an absence of symmetry. The dictionary of the French Orthodontic Society (ODF) puts the emphasis on the Greek root "dys" (bad) that indicates a slight disproportion of the face or of an organ. Therefore, the reasoning behind orthopedic dentofacial treatments logically leads us to assess the face of our patients, even though the fine line between what is considered pathological or not remains uncertain.

The methods for evaluating cutaneous and osseous tissues use norms for esthetic evaluation as well as for clinical diagnosis. We first evaluate the face of the patient but what are the reference norms that are used to support the extraoral examination? The esthetic assessment relies on pre-established criteria: facial norms based on cutaneous reference points. These proportions will not be discussed in this first section, but we should keep in mind that they are omnipresent in the work of great artists (The Vitruvian Man by Da Vinci) and in orthodontic values or mathematics (in 1981, Ricketts found the "golden proportion", the ratio between the different parts of the face, and of the mandible). The approach used here to assess dyssymmetries was arbitrarily vertical, but the saggital and transverse abnormalities are practically always combined and concurrent.

Therefore a defect of facial symmetry is most often associated with a malocclusion. In his article, Yamamoto found that there was a relation- ship between the type of malocclusion and the prevalence of dyssymmetries: $28 \%$ of patients in class I or class II and $40 \%$ of the groups in hyperdivergent class I or class II presented varying degrees of facial dyssymmetry. Dental, maxillary and cranial discrepancies as a whole, will leave an unavoidable and unmistakable signature on the face that the clinician can study. In the presence of a certain number of architectural discrepancies, the interpretation of the occlusal disorders will be more easily understood and the correlations with the underlying tissue will be even more justified. According to the diagrams of Duchateaux et al. the face can present different types of dyssymmetries:

- differences in the vertical positions of the cutaneous lines,

- differences of proportions,

- a curved or broken plane of symmetry that no longer matches the median saggital plane.

In the figure above to the right (Fig. 1): two halves of a face demonstrate the differences of proportions. However, we have to differentiate between a hypodevelopment and a lateral hyperdevelopment, since the two present very often together. These disproportions can affect all areas of the face as well as the vault and/or the base of the skull. In his anthropological study of the medieval population of the Val-de-Marne, Paleolios found a significant number of dyssymmetries $16 \%$ of the population) of the lower third of the face, showing a difference of the length of the mandibular rami. These discrepancies can appear very early and are often due to asymmetry of the 
base of the skull that causes a malocclusion accompanied by problems of dental intercuspation. The dyssymmetry can remain stable when cranial synchondroses close early, or appear later as major facial asymmetries become developmental. Then, we are referring to mandibular laterodysmorphias that Salagnac defines as abnormalities of dimension and volume that affect one or both sides of the mandible and are frequently accompanied by a lateral mandibular position in relationship to the plane of symmetry of the face.

It is widely agreed that mandibular laterodysmorphias are classified into two groups: functional and structural anomalies.

- Anomalies of lateral positioning of the mandible, that is a lateral movement measured by a median facial plane and naturally in relation to the maxilla, even though the dimensions of the mandible remain normal and subnormal.

- Osseous laterognathias that are also osseous abnormalities, are grouped into two categories: anomalies of dimension, shape or mandibular volume and secondly, asymmetries of the base of the skull that gives rise to an equally asymmetric location of the mandible. Of course, both of these can be present concurrently.

In the same vein, Planas mentioned crossbites in the deciduous dentition in his theory on neuro-occlusal rehabilitation "with early diagnosis, these atrophies are the easiest to treat but if they are ignored, the practitioner comes up against problems linked to the risk of the onset of irreversible dystrophies of the oss- eous bases." When centric occlusion during maximal intercuspation becomes impossible, the mandible settles to a lower position at the expense of a right or left lateral deviation. Once this becomes a habit and the masticatory process is established, the whole system will adapt to this pathological function, as the TMJ opposite the masticating side is more stimulated, this entails a more significant development of this side of the mandible with deviation of the midline of the incisors and of the point of the chin.

It is accepted that in the context of the face and more generally in nature, perfect symmetry is an illusion. However, we are all attempting to quantify defects in symmetry and by using therapeutic procedures, to reestablish symmetry of the dental arches, maxillary symmetry and symmetrical functioning of the jaws.

Orthodontics can intervene by decompensating minor forms of dissymmetry: we are all trying to distinguish between acceptable dyssymmetries that are considered normal and pathological asymmetries. The objective of diagnosis is to identify the source and extent of the defect of symmetry and to precisely determine possible therapeutic treatments.

\section{2 - DIAGNOSIS}

The diagnosis of mandibular dysmorphias is based on a methodical study of the patient's medical history, on the clinical examination and complementary diagnostic items that are mainly comprised of radiological 
studies, photographs and dental casts. Laterodysmorphias have various etiologies, either structural or functional, and must be detected as early as possible in order to begin an individualized therapeutic treatment.

\section{2 - 1 - Patient medical history}

The patient's medical history is the preamble to the clinical examination; this vital and indispensable examination makes it possible to evaluate the patients based on their history, as well as on their familial, environmental and psychological profile. How patients perceive their self-image is extremely important for the therapeutic treatment plan. In addition, we have to elicit important facts such as the age when it first presented, previous trauma or the developmental aspect of the dyssymmetry, that can all be demonstrated by anterior facial photographs.

\section{2 - 2 - Clinical examination}

The clinical examination of mandibular laterdysmorphias should not be limited to an occlusal examination, it must be performed from a global perspective in order to discern the etiopathology of the anomaly so as to provide an appropriate therapeutic response.

\subsubsection{Extraoral examination}

The assessment should begin with a general examination of the patient, by evaluating the overall posture: from the head, neck, shoulders, pelvis to the feet. This makes it possible to distinguish a facial asymmetry from a general asymmetry. By solely examining the face, the practitioner cannot precisely determine the site or the extent of the dissymmetry that is often hidden by cutaneous tissue.

Peck and Peck point out that "asymmetries" of the face have always existed without ever being considered unesthetic. Therefore, a clinical evaluation is linked to the esthetic sensibility of the observer. From a frontal view, reference systems of vertical symmetry (mid-saggital plane) and hortizontal symmetry (cutaneous Frankfort horizontal plane, bicommissural line, bigonial line, submental line,...) have been established in order to better visualize the divergences.

Bischara and Burstone recommend an overhead view so as to highlight abnormalities due to asymmetric unilateral mandibular development.

An examination of the musculature, of the dysfunctions and of the parafunctions is performed as with any dysmorphia, in search of a possible etiology.

An assessment of the chin in the rest position, with lips closed, teeth clenched and while smiling could reveal a deviation in the case of functional jaw shift.

\subsubsection{Intraoral examination}

The intraoral examination is a decisive element in the diagnosis of mandibular dyssymmetry and makes it possible for the practitioner to accurately identify anomalies at the level of the dental arches and to visualize the occlusal relationships. 
This examination precisely determines the relationships between the maxillary and mandibular interincisal midlines as well as the position of these midlines in relation to facial symmetry.

It compares the transverse dimensions of the maxillary and mandibular arches thus making it possible to demonstrate an anomaly of position or of dimension.

When asymmetric wear of the occlusal surfaces is diagnosed it could indicate unilateral mastication (Planas' theory).

When screening for mandibular dissymmetry, a study of the occlusal relationships must be undertaken in different positions in the following sequence:

- In maximum intercuspation occlusion (CO),

- In centric relation occlusion (CR),

- In rest position and mouth slightly open.

This examination will provide evidence for either a structural or functional cause of the dysmorphia.

Osseous abnormalities or mandibular laterognathias are permanent and the position of the interincisal midline is identical in the different occlusal positions whereas with abnormalities of position, referred to as lateral sliding or mandibular lateral deviation, the deviation of the mid-incisors in CO disappears in rest position and in $\mathrm{CR}$.

It does not take much to bring on this comfortable lateral occlusion: prematurity in the path of closure or premature canine contact can cause this modification in mandibular movements.

When lateral deviation goes untreated, it can lead to the creation of a structural lateral jaw shift.
An examination of the path of opening and closing can show a lateral deviation during clenching or unclenching of the jaw.

\section{2 - 3 - Supplementary examinations}

\subsubsection{Photographic examination}

- Extraoral,

- frontal with teeth closed, in rest position and while smiling naturally in a seated position,

- right and left profile,

- from above (overhead),

- from below (low angle),

- intraoral,

- in CO frontal, right and left profile,

- in CR.

\subsubsection{Examination using plaster casts}

According to Izard, models must be done because even when it is not apparent, it can quantify the dental dyssymmetry. Mounting the models on a semi-adjustable articulator situates the arches in correct relation to face and allows the practitioner to visualize possible rotations in relation to basicranial reference points.

\subsubsection{Radiological examination}

This examination can provide evidence of skeletal dyssymmetry.

- Orthopantomogram (Fig. 6): as an unscrambling examination, it makes it possible to highlight a problem of symmetry by comparing the two halves of the mandible in relation to their shape and their dimension, volume and length of the heads and condylar necks, in relation to the 


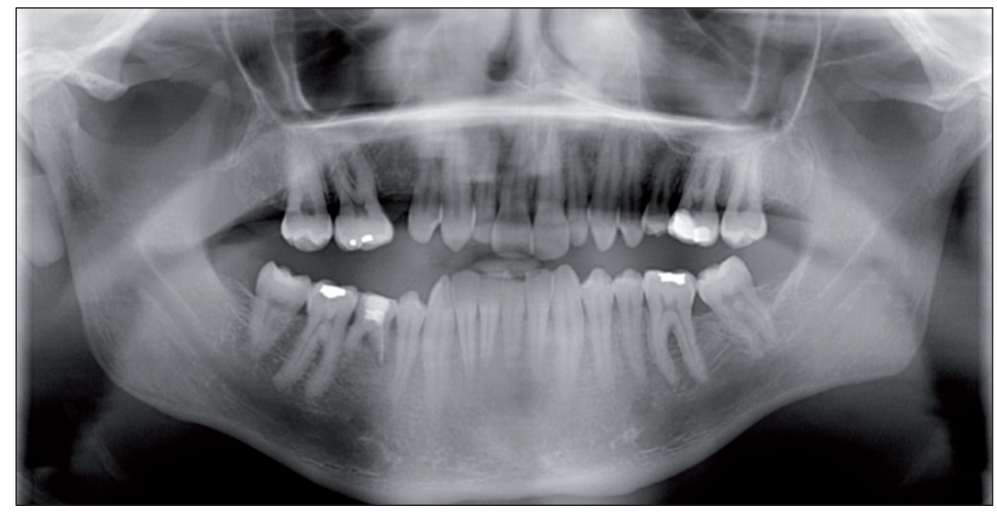

Figure 6

Orthopantomogram result. Mr. B. Case of Dr. MERLY J.C.

antegonial notch, the distance between the apex of the molars and the inferior border of the mandible.

- AP head film of the face (Fig. 7): preferably, it is taken in rest position to distinguish a structural dyssymmetry from a functional dyssymmetry, based on whether

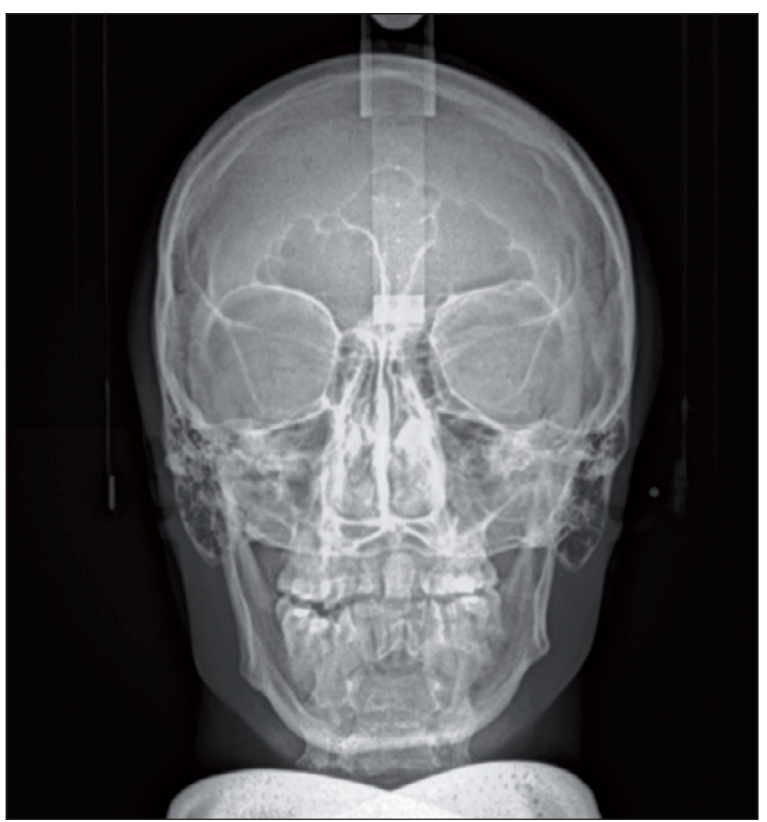

Figure 7

Complete vertical osteotomy tract. the mandible is symmetrical or not. This is the examination of choice for visualizing the two halves of the mandible in relationship to the midvertical axis of the face, and in relationship to the other structures.

- Lateral head film (Fig. 8): even though it does not allow for the

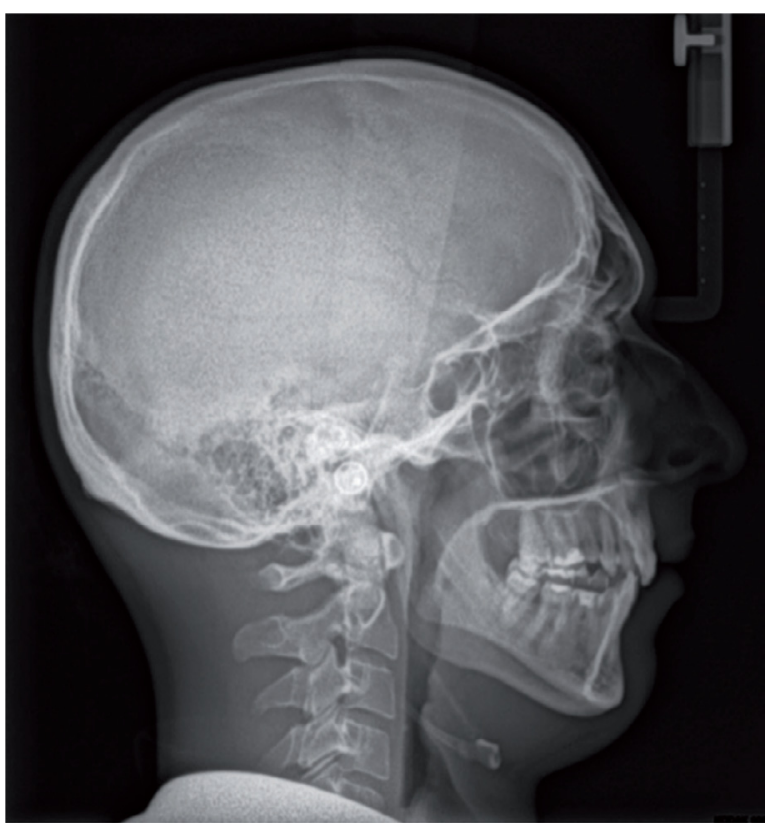

Figure 8

Lateral head film. Mr. B. Case of Dr. Merly J.C. 
- We are including these summary fact sheets that will help practitioners clearly differentiate these anomalies in their daily practices.

\section{(LATERAL) FUNCTIONAL MANDIBULAR JAW SHIFT}

- Midline deviation in CO, alignment of the midlines in $\mathrm{CR}$ or in rest position

- Non-developmental nature and, if detected early, reversible.

- Etiopathogenesis (or Etiopathology) : a prematurity in the path of closure often located in the primary canine area.

\section{Clinical signs:}

- Facial extraoral: facial dyssymmetry (of the chin) in CO that corrects itself in CR.

- Intraoral: in CO, the PII (the midlines) are deviated because of a prematurity and are coincident in CR. The maxilla often presents in crossbite due to insufficient growth.
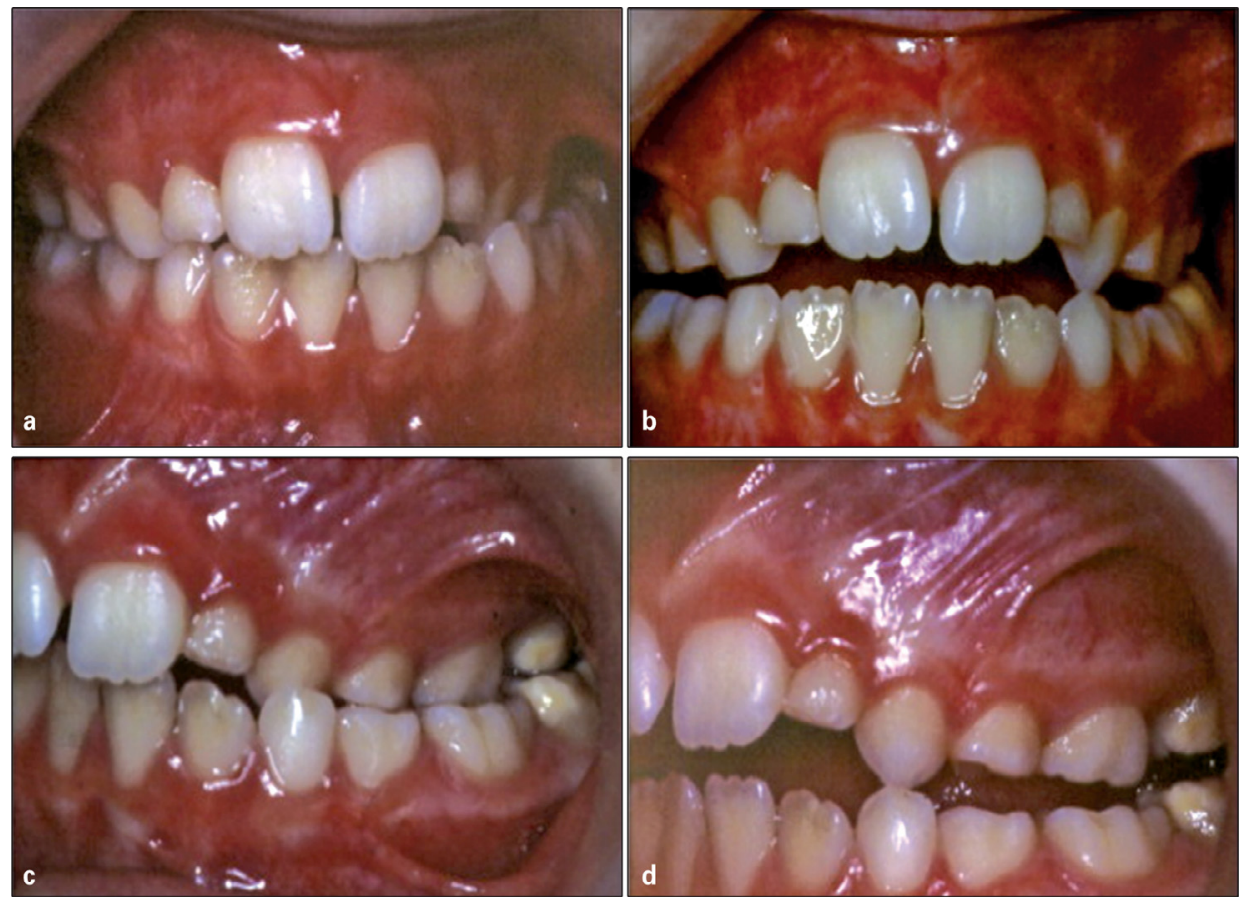

Figure 9

a .\& b.: Maximum occlusal intercuspation (CO)

c. \& $d$. : Occlusion in centric relation (CR)

Case of Doctor LE GALL M. 


\section{CONDYLAR HYPERPLASIA}

- Rare and benign, characterized by hyperplasia linked to hyperactivity of the condylar cartilage.

- Deviation of the midlines in CO, CR and rest position.

- Etiopathogenesis (or Etiopathology): traumatic, vascular, pathological neural development, local or regional inflammation.

- Developmental in nature, is discovered usually beginning between 10 and 12 years of age, irreversible.

\section{Clinical signs:}

Extra-oral:

- Face: dissymmetry of the lower face, a reduction with convexity of the inferior border of the mandible which extends from the angle of the mandible to the chin, a drooping of the commissure of the lips on the side of the condylar hyperplasia.

- Profile: on the side of the condylar hyperplasia: a prominence of the chin, a reduction of the inferior border of the mandible and the angle of the mandible. Intraoral:

- The occlusion can present in various ways: it can be mildly affected or not at all, presenting a unilateral infraocclusion, or an inverted occlusion. Radiographic:

- Orthopantomogram: one condylar head that is larger with a more elongated neck on the side of the condylar hyperplasia. A global convexity along the inferior border of the mandible associated with the lack of an antegonial notch. An increase in the distance between the apices of the molars and the inferior border of the mandible.

- Lateral head film: dissymmetry between the two condyles, a vertical differential in the entire (angle of the mandible, inferior border of the mandible).

- AP head film: mandibular dissymmetry by a reduction of the angle of the mandible and the inferior border of the mandible on one side, deviation of the mandibular symphysis, canting of the occlusal plane or compensation of the mandibular incisors.

\section{CONDYLAR HYPOPLASIA}

Clinical signs: Inverse of condylar hyperplasia.

Radiographic examination:

- Lateral head film: dyssymmetry of the two condyles, vertical differential of the entire mandible (angle of the mandible, inferior border of the mandible).

- AP head film: mandibular dyssymmetry by a reduction of the angle of the mandible and the inferior border of the mandible of the healthy side, deviation of the mandibular symphysis, canting of the occlusal plane or compensation of the mandibular incisors. 
study of mandibular symmetry in its transverse sense, this film does make it possible to assess the situation of the angle of the mandible, saggital misalignment of one ascending ramus in relationship to the other and to look for an unbroken double-contour image of the horizontal and ascending rami of the mandible that might be a panthognomonic for mandibular laterognathias according to Chouquin.

- A de Bouvet axial radiography: this is the method of choice for determining the shape and the dimensions of the mandible and more particularly of the condyles. It must be taken in CR so as to avoid errors during lateral deviation.

- Electromyography: little used muscular examination, that provides precise information concerning the muscular etiology of the laterodysmorphia.

Other examinations such as a scanner, bone scan, axiography or stereolithography, can provide further proof or precisely determine the etiology of the mandibular dyssymmetry.

In cases of mandibular laterodysmorphias, two pathologies with structural causes (condylar hyperplasia and condylar hypoplasia) and functional jaw shift are most frequently encountered in our offices.

\section{CONCLUSION}

Mandibular dyssymmetry varies. The most impressive forms are associated with major syndromes. The age of the patient, the extent of the development, the clinical examina- tion, complementary examinations allow the practitioner to render an exact diagnosis. This is fundamental for establishing a therapeutic treatment plan best suited to the patient.

\section{REFERENCES FOR CONSULTATION}

AKNIN JJ. La Croissance cranio-faciale ; SID 2007.

BAZERT C., BOILEAU M-J., DE BRONDEAU F., DORIGNAC D., EL AMRANI-DARQUÉ K., GARNIER E., PERRIER M., PUJOL A. et ROTENBERG M., Approche étiopathogénique et clinique des dyssymétries, Orthod Fr 2002;73:125-178.

BETTEGA G., MORAND B., LEBEAU J., RAPHAEL B., Les altérations morphologiques au cours des syndromes oto-mandibulaires, Ann Chir Plast Esthét 2001;46:495-506.

CHEONG Y., LO L. J. Facial asymmetry: Etiologie, Evolution and Management, Chang Gung Med J 2011;34(4).

$\mathrm{CHO} \mathrm{K} \mathrm{H}$. Dyssymétries cranio-faciales et dysmorphoses. Analyse architecturale craniofaciale des populations médiévales de la Queue-en-Brie (Val-de-Marne), ThÖse de doctorat, Muséum d histoire naturelle, Vol. 1, 451 p., 2005.

COHEN M. M. Perspective on craniofacial asymetry, Int J Oral Maxillofac Surg 1995;24:2-4.

DELAIRE J., JACQUEMAIRE D. L hypercondylie mandibulaire, Rev Stomatol Chir Maxillofac 1983;84:5-10. 
DUCHATEAUX C. L., VION P., FERRE J. C., CHOQUIN Y., Le diagnostic des dissymétries squelettiques faciales, Orthod Fr 1974;45:7-26.

DUCREUX M., DUVERNAY A., MALKA G., TROUILLOUD P., TROST O., Fentes labiomaxillo-palatines:

aspects morphologiques et embryologiques, Rev Orthop Dento Faciale 2011;45(3):263269.

DUFFIT C., BAGGIO E., RUBAN J-M., BUEBERD A., HERMIER M., L anophtalmie foetale. ProblÒme du diagnostic anténatal : A propos d'un cas, J Fr Ophtalmol 1999:22(9),966-969. Esthétique du visage, EMC consulte, Vol. 46, No. 5, p:410-423.

HARAGUCHI S., IGUCHI Y., TAKADA K., Asymmetry of the face in orthodontic patients, Angle Orthodontics 2008;78(3).

LASPOS C P., KYRKANIDES S., TALLENTS R. H., MOSS M. E., SUBTELNY J. D., Mandibular and Maxillary asymetry in individuals with Unilateral Cleft Lip and Palate, Cleft Palate Craniofacial Journal 1997,34(3);232-239.

LASPOS C P., KYRKANIDES S., TALLENTS R. H., MOSS M. E., SUBTELNY J. D., Mandibular asymmetry in Noncleft and Unilteral Cleft Lip an Palate Individuals, Cleft Palate Craniofacial Journal, 1997,34 (5);410-416.

NETHERWAY D.J., ABBOT A.H., GULAMHUSEINWALA N. McGAUGHLIN M.B., ANDEERSON P.J., TOPWNSEND G.C., DAVID D.J., Three dimensional Computed Tomography Cephalometry of Plagiocephaly: Asymmetry and shape Analysis, Cleft Palate Craniofacial Journal 2006;43(2);201-210.

SALAGNAC J.-M., Latérodysmorphose mandibulaire EMC (Elsevier SAS, Paris), Odontologie/orthopédie dento faciale, 23-472-F-10, 2006.

TALMANT J.C., TALMANT J. Ch., LUMINEAU J. P., Aspect morpho-fonctionnel et répercussion sur la croissance. Rev Orthop Dento Faciale 2011;45(3):263-269.

VIG P. S., HEWITT A. B. Asymmetry of the human facial skeleton, Hum Biol 1975;45(2). TAKANO-YAMAMOTO T., KURODA S., Diagnostic et traitement de patients adultes présentant une dyssymétrie faciale, Orthod Fr 2009;80:313-319.

LE GALL M., PHILIP C., BANDON D., Les anomalies maxillomandibulaires du sens transversal chez l'enfant, Archives pédiatriques 2009;16:209-213.

Bien programme mä̈trise 3 brionne11mars.indd 152 16/04/13 10:09 